\title{
Proptosis y oftalmoparesia oculomotora completa secundaria a una arteria trigeminal persistente
}

\section{Proptosis and complete oculomotor ophthalmoparesis due to a persistent trigeminal artery}

\author{
Glenda Espinosa-Barberi ${ }^{1,2 *}$, Francisco Medina-Rivero ${ }^{1}$, Pedro Saura-Lorente ${ }^{3}$ y Manuel Maynar-Moliner ${ }^{4}$ \\ ${ }^{1}$ Departamento de Oftalmología, Hospital Universitario Doctor Negrin, Las Palmas de Gran Canaria; ${ }^{2}$ Departmento de postgrado y doctorado, \\ Universidad de Las Palmas de Gran Canaria, Las Palmas de Gran Canaria; ${ }^{3}$ Departmento de Neuroradiología, Hospital La Paz, Madrid; \\ ${ }^{4}$ Departmento de Ciencias Médicas y Quirúrgicas, Universidad de Las Palmas de Gran Canaria, Las Palmas de Gran Canaria. España
}

\begin{abstract}
Resumen
La arteria trigeminal persistente es una anomalía vascular poco común que se presenta como una comunicación entre la arteria carótida interna y el sistema vertebrobasilar. Esta anastomosis es importante debido a que asegura el correcto aporte sanguíneo del cerebro durante el desarrollo embrionario, que es la etapa más crítica. El diagnóstico, en la mayor parte de los casos, es un hallazgo accidental. En el $25 \%$ de los casos, la arteria trigeminal persistente se relaciona con algunas enfermedades cerebrales de tipo vascular, especialmente con aneurismas intracraneales, mientras que la repercusión neurooftalmológica no es frecuente. Se reporta el caso clínico de un paciente varón que desarrolla una proptosis, así como una oftalmoparesia completa del tercer par craneal con una variante anatómica de la normalidad, que consiste en una arteria trigeminal persistente detectada mediante resonancia magnética.
\end{abstract}

Palabras clave: Nervio oculomotor. Proptosis. Angiografía. Diplopía. Oftalmoplejía.

\begin{abstract}
The persistent trigeminal artery (PTA) is a rare vascular anomaly which represents a persistent communication between the internal carotid artery and vertebrobasilar system. This anastomosis is important because it provides the main source of blood supply during the development of the embryonic brain in its most critical stage. The diagnosis, in the majority of cases, concerns an incidental finding. The $25 \%$ of the PTAs are linked to some vascular brain diseases, especially with intracranial aneurysms, and neurophthalmologic involvement is not frequent. This case reports a male patient who developed proptosis and a complete ophthalmoparesis of the third cranial nerve with an anatomical variant of the normality consisting of a PTA detected by the magnetic resonance imaging.
\end{abstract}

Key words: Oculomotor nerve. Proptosis. Angiography. Diplopia. Ophthalmoplegia.

Correspondencia:

*Glenda Espinosa-Barberi

Hospital Universitario Doctor Negrin

Plaza Barranco de la Ballena, $s / n$

C.P. 35010, Las Palmas de Gran Canarias, España

Fecha de recepción: 19-06-2017

Fecha de aceptación: 12-03-2018

E-mail: glenda_eb@ hotmail.com

DOI: 10.24875/RMO.M19000094
Disponible en internet: 01-11-2019 Rev Mex Oftalmol. 2019;93(6):308-311

www.rmo.com.mx 0187-4519/@ 2018 Sociedad Mexicana de Oftalmología. Publicado por Permanyer México. Este es un artículo Open Access bajo la licencia CC BY-NC-ND (http://creativecommons.org/licenses/by-nc-nd/4.0/). 


\section{Introducción}

En la etapa embrionaria, la circulación posterior, representada por el sistema vertebrobasilar primitivo, depende en gran medida de la anastomosis establecida con la carótida interna primitiva. Una de estas anastomosis está representada por la arteria trigeminal persistente (ATP) (representa el $85 \%$ de los casos de vasculatura permanente en la edad adulta). Se designa así debido a su vínculo con el nervio craneal más cercano, y conecta la porción cavernosa de la arteria carótida interna $(\mathrm{ACl})$ con el sistema basilar. Esta anastomosis es importante porque proporciona la principal fuente de suministro de sangre durante el desarrollo del cerebro embrionario en su etapa más crítica ${ }^{1-3}$.

Se origina durante la cuarta semana de vida intrauterina y sufre un proceso de regresión en la octava semana, período en el que se garantiza el suministro de sangre correcto hacia la fosa posterior ${ }^{1,3}$.

Existen varias clasificaciones anatómicas de la ATP de acuerdo con su ruta, que anteriormente llevaban el nombre de Saltzman. La más importante desde la perspectiva clínica se origina en el seno cavernoso, y fluye proximal al nervio trigémino sensorial, lateral a la silla turca (Saltzman tipo 2). Esta variante a menudo se ha relacionado con enfermedades cerebrovasculares isquémicas, neuralgia y parálisis del trigémino, y paresia o parálisis oculomotora, mientras que la variante medial (menos frecuente que la anterior y llamada Saltzman tipo 1) se ha relacionado con síntomas causados por síndromes de "robo" de fosa posterior debido al hecho de que está conectada a las arterias comunicantes posteriores basilares e hipoplásicas ${ }^{2,3}$.

El diagnóstico, en la mayoría de los casos, se debe a un hallazgo incidental en la vasculatura cerebral a través de la neuroimagen, que se encuentra en $0.1 \%$ a $0.6 \%$ de las angiografías cerebrales realizadas. Sin embargo, hay casos reportados en los que se ha propuesto su etiología directa con varios procesos patológicos como las compresiones neurovasculares y los síntomas vertebrobasilares ${ }^{3-8}$. El $25 \%$ de las arterias trigeminales persistentes están vinculadas a algunas enfermedades cerebrales vasculares, especialmente aneurismas intracraneales, que se encuentran con frecuencia en la bifurcación de la carótida interna ${ }^{2,6,8}$.

Presentamos un informe de caso sobre un paciente masculino con esta patología que debutó con signos oftalmológicos que, aunque están descritos en la literatura, no cuentan con casos recientes publicados.

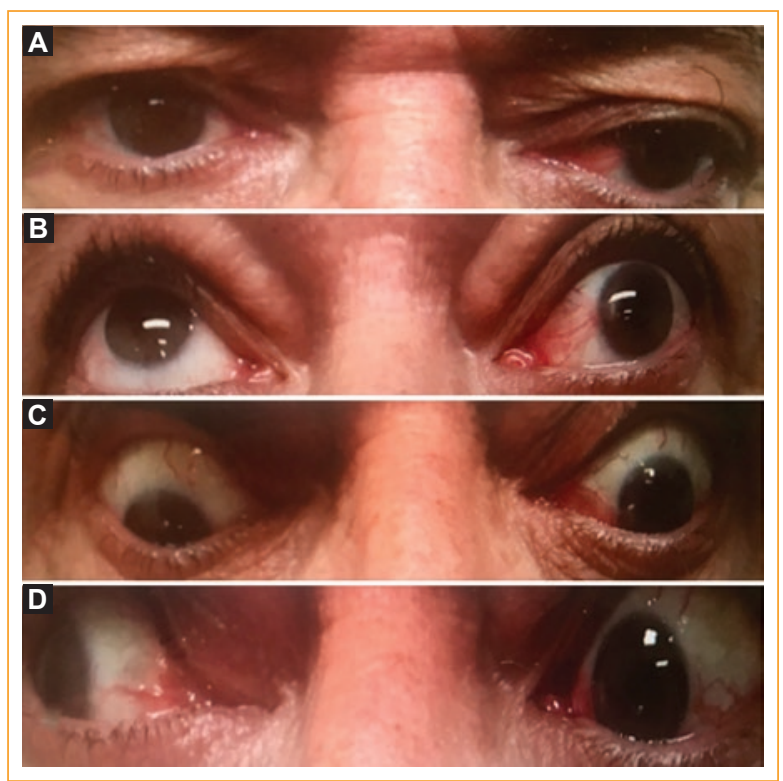

Figura 1. Fotografía externa. A: hiperemia conjuntival, proptosis, ptosis y exotropía del ojo izquierdo. B: limitación a la supraversión. C: limitación a la infraversión. D: limitación a la aducción.

\section{Informe de caso}

Un paciente masculino de 63 años de edad, previamente sano, fue derivado con un historial de desarrollo de proptosis de 4 años en el ojo izquierdo (OI), acompañada de diplopía, quemosis conjuntival y ptosis palpebral (Fig. 1). En las exploraciones oftalmológicas, la agudeza visual era de 20/40 en el ojo derecho (OD) y 20/63 en el OI, además de una limitación en la motilidad oculomotora a la aducción, supraversión e infraversión (Fig. 1). En el segmento anterior, se observó midriasis hiperreactiva. El fondo ocular era normal en ambos ojos, pero el campo visual anormal del OI mostró un gran escotoma generalizado.

Se detectó una lesión en la densidad de los tejidos blandos en la tomografía axial computarizada (TAC) de las órbitas. La lesión ocupaba el ápice orbitario, el agujero óptico y el seno cavernoso izquierdo, por lo que se solicitó una resonancia magnética que mostró una variante anatómica de ATP desde la arteria carótida izquierda hasta el tercio medio de la arteria basilar que genera un aumento del tamaño de la arteria carótida izquierda del mismo lado secundario a un incremento en el flujo de salida, sin que se identifiquen posibles aneurismas ni alteraciones inflamatorias a nivel del seno cavernoso (Fig. 2).

Los potenciales evocados visuales mostraron un retraso en la conducción de la vía visual retrobulbar izquierda, con amplitud disminuida. 


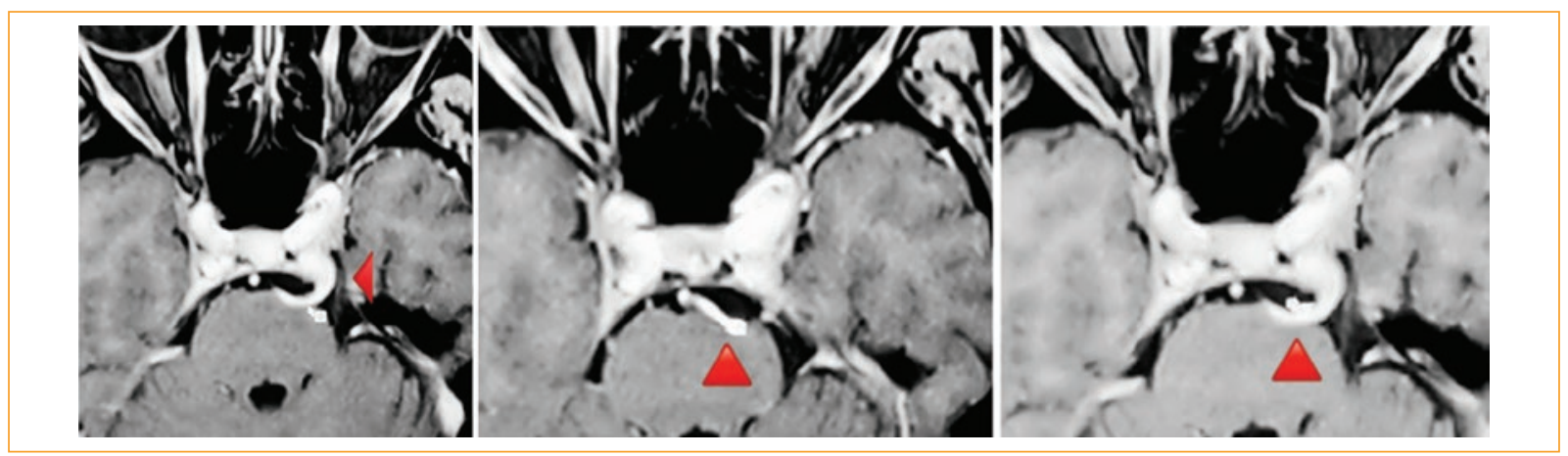

Figura 2. Resonancia magnética de cráneo. Secuencia axial ponderada en T1 que muestra una imagen tubular izquierda correspondiente a la arteria trigeminal persistente que viaja desde la arteria carótida izquierda hasta el tercio medio de la arteria basilar.

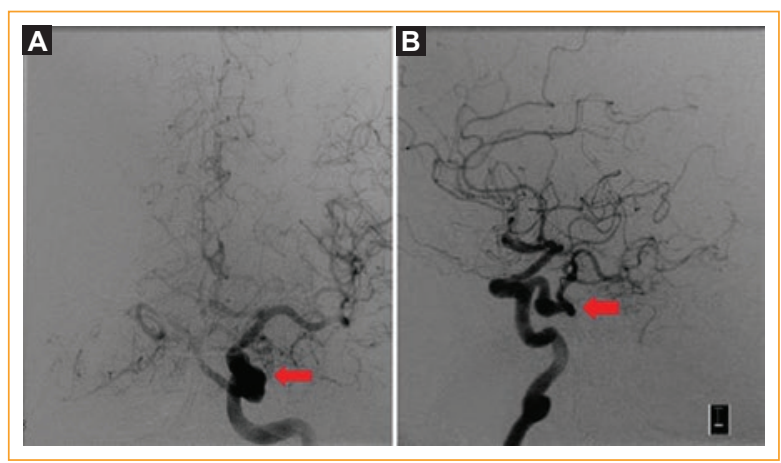

Figura 3. A,B: Angiografía selectiva izquierda: origen de la arteria trigeminal persistente y su ruta hacia la arteria basilar.

Una vez que se confirmó la causa de la oftalmoparesia completa del tercer nervio craneal debido a compresión de la ATP a nivel mesencefálico, se decidió realizar una angiografía carotídea selectiva, donde se observó alargamiento y tortuosidad severa de los troncos supraaórticos con estenosis severa en la salida de la arteria vertebral izquierda; lo que hace imposible su cateterización (Fig. 3).

\section{Discusión}

La ATP es una anastomosis del sistema cerebral vascular embrionario, que fluye entre la $\mathrm{ACl}$ y la arteria basilar y, por lo tanto, conecta la circulación anterior con la posterior. Su presencia altera la organización habitual de los nervios craneales dentro del seno cavernoso; en consecuencia, los nervios oculomotores podrían desplazarse en cualquier dirección ${ }^{1-3}$.

Generalmente se presenta de manera asintomática y se identifica de forma incidental en ciertos estudios angiográficos realizados por otras razones. Dentro del cuadro clínico, la afectación de los nervios oftálmicos se encuentra en tercer lugar, junto con la neuralgia del trigémino, debido a compresión. Dentro del compromiso oculomotor, el nervio VI es el más afectado (hasta 4 de 6 casos), seguido del III nervio (como en nuestro caso) $^{9}$.

El nervio oculomotor (nervio III) se origina en los núcleos mesencefálicos. Antes de su entrada en la órbita, penetra a través de la pared lateral del seno cavernoso junto con el nervio patético, el nervio abducens y la primera rama del nervio trigémino. La compresión nerviosa podría producirse a nivel del mesencéfalo y/o a nivel del seno cavernoso $0^{9,10}$.

Sus lesiones se manifiestan con diplopía binocular, ptosis, parálisis de la elevación y alteraciones de la abducción; si se debiera a compresión, la inervación parasimpática del músculo ciliar se vería afectada, lo que daría lugar a midriasis no reactiva ${ }^{9,10}$. Nuestro paciente es compatible con una oftalmoparesia izquierda completa del tercer nervio craneal.

La parálisis del tercer nervio se clasifica como infranuclear y supranuclear. La primera es adquirida y causada en su mayor parte por enfermedades vasculares, malformaciones vasculares y tumores, entre otros. Como la arteria trigeminal se asocia frecuentemente con alteraciones de la vasculatura cerebral, puede producir oftalmoparesia debido a compresión, hemorragia, aneurismas e incluso fístulas, en cuyo caso puede causar dolor ocular, quemosis, proptosis y oftalmoplejia. En el caso presentado, las alteraciones son producidas por la propia arteria, ya que presenta una dilatación fusiforme significativa.

Todos los pacientes deben ser evaluados neurorradiológicamente. La primera prueba debe ser una TAC, que permitirá visualizar la arteria trigeminal y, posteriormente, una resonancia magnética angiográfica 0 una angiografía para confirmar el diagnóstico ${ }^{1,3}$. Si el 
paciente presentara en cualquier momento cefalea severa, además de los otros síntomas que lo acompañan, sería indicativo de un caso ictal o accidente cerebrovascular, por lo que las medidas a realizar serían invasivas.

En conclusión, la ATP es una variante anatómica rara, su diagnóstico en la mayoría de los casos suele ser incidental porque no presenta manifestaciones clínicas, y si presentara alguna, el cuadro más común sería cerebrovascular. La parálisis oculomotora ocuparía, de esta manera, el tercer lugar en frecuencia. Debido a esto, es una patología que debe tomarse en cuenta cada vez que se realice un diagnóstico diferencial en respuesta a estas alteraciones. Los pacientes deben ser evaluados neurorradiológicamente.

En caso de realizar el diagnóstico, se requiere un estudio anatomo-vascular completo debido a su alta relación con alteraciones de la morfología vascular-cerebral y aneurismas que podrían requerir tratamiento endovascular/neuroquirúrgico en caso de que el cuadro se vuelva sintomático.

\section{Agracedimientos}

Un agradecimiento especial al Sr. Miguel Froján García, Licenciado en Filología Inglesa en la Universidad de Santiago de Compostela (certificado de capacidad educativa y certificado en adultos que enseñan inglés), quien contribuyó en la traducción del texto presentado, así como en la corrección gramatical.

\section{Responsabilidades éticas}

Este manuscrito ha sido evaluado favorablemente por el comité de ética e investigación del Hospital Universitario Doctor Negrin, con el siguiente código de aprobación: 170072.

Protección de personas y animales. Los autores declaran que los procedimientos seguidos se conformaron a las normas éticas del comité de experimentación humana responsable y de acuerdo con la Asociación Médica Mundial y la Declaración de Helsinki.

Confidencialidad de los datos. Los autores declaran que siguieron los protocolos de su centro de trabajo sobre la publicación de datos de pacientes.

Derecho a la privacidad y consentimiento informado. Los autores han obtenido el consentimiento informado de los pacientes y/o sujetos referidos en el artículo. Este documento obra en poder del autor de correspondencia.

\section{Financiación}

No se recibieron apoyos de ningún tipo para realizar este estudio/artículo.

\section{Conflicto de intereses}

Los autores declaran no tener ningún conflicto de intereses.

\section{Referencias}

1. Valverde-Lavirgen $\mathrm{S}$, Núñez GS, Duque $\mathrm{CH}$, et al. La arteria trigeminal persistente, una variante que debe reconocerse a pesar de su infrecuencia. Rev Neurol. 2007;44:625-7.

2. Luis $E T$, Jorge $A R$, Arias $D E$, et al. Arteria trigeminal persistente: reporte de tres casos. Rev Neuropsiquiatr. 2014;77:110-5.

3. Cerra GA, Paternina JG, Salazar LM, et al. Implicaciones de la arteria trigeminal persistente. Rev Cienc Biomed. 2013;4:147-51.

4. Perals LF, Artero JM, Estrada LF, et al. Aneurisma intracavernoso asociado a arteria trigeminal primitiva persistente. Neurocirugía. 1997; 8:36-40.

5. Uchino A, Kato A, Takase Y, Kudo S. Persistent trigeminal artery variants detected by MR angiography. Eur Radiol. 2000;10:1801-4.

6. Olivares J, Verdegay GA. La arteria trigeminal persistente y VI par craneal aislado. Rev Neurol. 2007;44:685-6.

7. Agrawal D, Mahapatra AK, Mishra NK. Fusiform Aneurysm of a persistent trigeminal artery. J Clin Neurosci. 2005;12:500-3.

8. Suttner N, Mura J, Tedeschi $\mathrm{H}$, et al. Persistent trigeminal artery: a unique anatomic specimen-Analysis and therapeutic implications. Neurosurgery. 2000;47:428-33.

9. Kalidindi RS, Balen F, Hassan A, Al-Din A. Persistent trigeminal artery presenting as intermittent isolated sixth nerve palsy. Clin Radiol. 2005; 60:515-9.

10. Adams RD, Victor M, Ropper AH. Trastornos de los movimientos oculares y de la función pupilar. En Principios de Neurología. $6^{\text {th }}$ ed. México DF: Mc Graw Hill-Interamericana; 1998. p. 227-48. 